\title{
Design and Implementation of Wireless Body Area Network using Physiological Parameters
}

\author{
Savita Fet, Dr. Shruti Vashist \\ Manav Rachna International University \\ savita.fet@mriu.edu.in, shruti.fet@mriu.edu.in
}

\begin{abstract}
Applications of e-Health deals with various physiological signals like body temperature, Blood Pressure, Electrocardiogram(ECG) captured by either wired networks or wireless sensor networks (WSN).ECG is one of the important parameter. A three lead based ECG acquisition system, body temperature; pulse rate monitoring system is presented in the paper below which can store data. The data can be sent wirelessly to the doctor for further analysis. The proposed design makes use of wearable sensors which will not be of discomfort to the patient. The patient can move around easily.
\end{abstract}

Keywords: Wireless Sensor Network, Physiological Parameter, ECG, Body Temperature

\section{Introduction}

It is very apparent for a person to visit hospital for routine checkup of temperature, blood pressure etc., but it become very arduos for elderly persons or patient's suffering from critical diseases like heart ailments etc. to visit the hospital on regular basis. They require quick and accurate line of treatment.Such patient requires continuous monitoring for long term health benefits. Electrocardiograph is one of the critical health indicators that benefits the patient in long term .If a person goes to hospital for routine checkup his/her body temperature and blood pressure checking is normal practice. Old age people and patients suffering from heart diseases (related diseases) need quick and accurate line of treatment. If such persons are put in continuous monitoring then it would be proven beneficial for them. Wireless body area network does the same [13]. Electrocardiograph is one of the critical health indicators that benefit the patient in long-term. ECG signal is a time-varying signal representing the electrical activity of the heart. It is an effective, non- invasive diagnostic tool for cardiac monitoring [1].

Xin Liu et al [6] proposed a power and area efficient electrocardiogram (ECG) acquisition and signal processing application sensor node for wireless body area networks (WBAN). This sensor node can accurately record and detect the QRS peaks of ECG waveform with high-frequency noise suppression. The proposed system was implemented in $0.18-\mathrm{mm}$ complementary metal-oxide-semiconductor technology with two chips: analog front end integrated circuit (IC) and digital application specific integrated circuit (ASIC). Therefore, this ECG sensor node is convenient for long-term monitoring of cardiovascular condition of patients, and was very suitable for on-body WBAN applications. Pandian et DOI: $10.14738 /$ jbemi.41.2800 
al. [6] gave a conceptual design of a wearable physiological monitoring system based on wireless sensor network to monitor physiological parameters like ECG, EMG, EEG, SaO2, body temperature, blood pressure, respiratory rate GSR and movement of the wearer. The acquired signals were pre-processed at each node at the sensor level and transmitted to the wearable data acquisition hardware (sink node) for further processing. It was then transmitted wirelessly to the remote monitoring station.

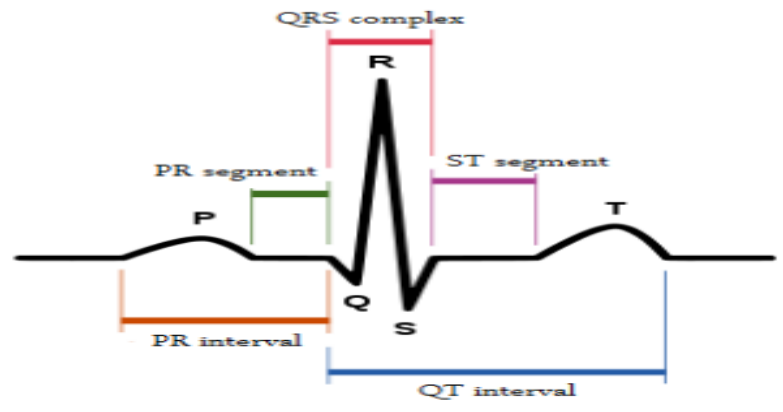

Figure 1: ECG signal

An ECG gives two major information's: Firstly, by measuring time intervals on the ECG, a doctor can determine how long the electrical wave takes to pass through the heart. Secondly, by measuring the amount of electrical activity passing through the heart muscle, a cardiologist may be able to find out if parts of the heart are too large or are overworked.

Traditionally, two categories of ECG monitors exist. The first is the one used in hospitals, and the other is the Holter telemonitoring system, which transmits ECG signals via telephone lines and can record data for more than 24 hours.[2]

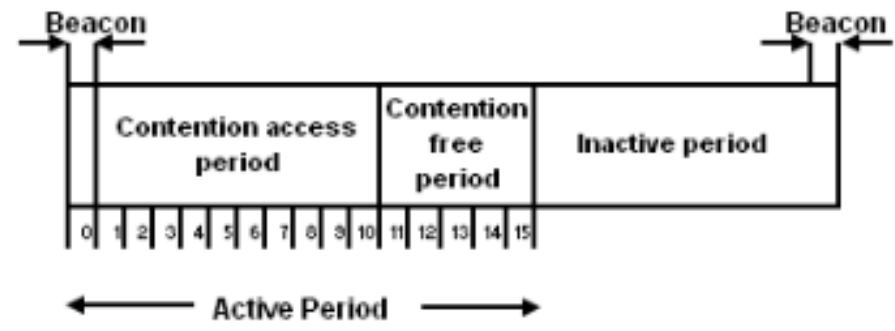

Figure 2: IEEE 802.15.4 standard defining Wireless PAN

IEEE 802.15.4 [11] is a low-power standard designed for low data rate applications. It offers three operational frequency bands: $868 \mathrm{MHz}, 915 \mathrm{MHz}$, and $2.4 \mathrm{GHz}$ bands. Basically two operation modes are present in IEEE 802.15.4 standard; they are beacon enabled and non-beacon enabled mode. A beacon frame is broadcasted periodically from the coordinator and it contains information, which is necessary to build the network [3]. In non becon mode data coordinator isn't require to transmit data [12].A network in which the (Personal Area Network)PAN coordinator does not transmit beacons is known as a Non beacon network.

Rui Pan Et.al [4] worked on WBAN based cable less ECG acquisition system in which 3-lead cableless electrocardiography (ECG) acquisition system was described. Measurements were obtained via a custom 
Savita Fet,; Design and Implementation of Wireless Body Area Network using Physiological Parameters. Journal of Biomedical Engineering and Medical Imaging, Volume 4, No 1, February (2017), pp 13-20

wireless network platform utilizing a TDMA-based MAC protocol supporting the star topology and a proprietary front-end ECG acquisition system.

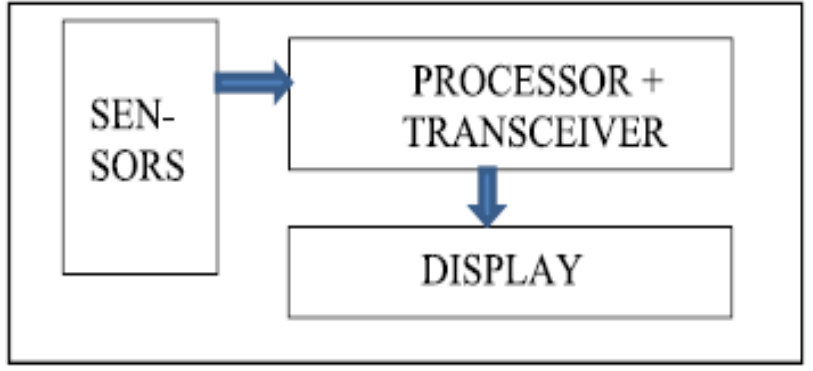

Figure 3.Block diagram representation of a simple wearable wireless sensing device

Figure 3 represents the block diagram of a simple wearable sensing device which shows that the transceivers take signals from all sensors mounted on the body and sends them to the processing unit. After processing, results are displayed. Navale et al [9] describes their system in which a patient will be carrying hardware having sensors and android phone application. The sensors will sense the body temperature and heart rate of patient and this data would be transferred to android smart phone via Bluetooth.

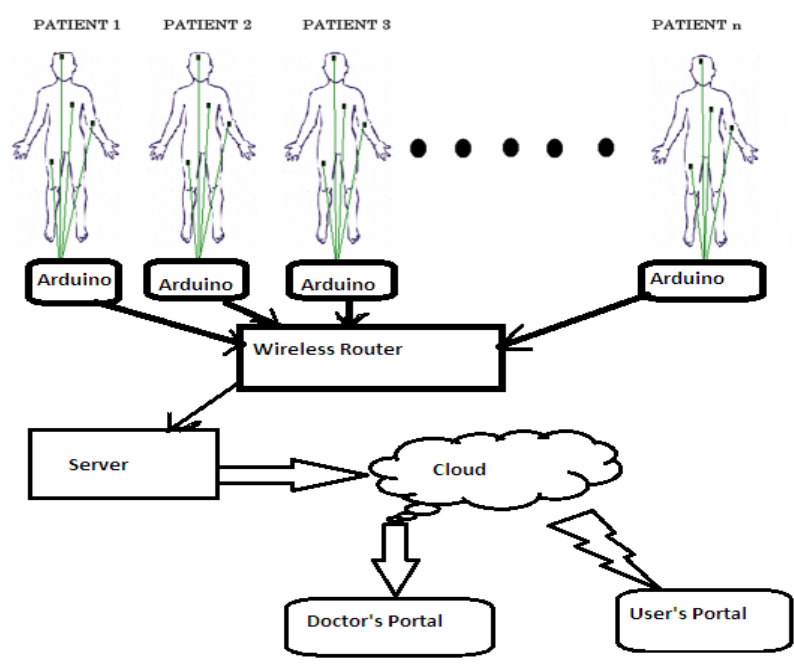

Figure 4: Actual representation of Wireless Body Area Network

Figure 4 represents the actual architecture of Wireless body area network which there illustrates $\mathrm{n}$ number of patients. They all are connected through arduino. Physiological data is taken from arduino and then data is sent to server. The data history can be saved on cloud and can be fetched as and when required .It can be sent to the doctor's and the user's portal for reference.

The paper is organized as follows: Section II represents the proposed architecture of Wireless Body Area Network. Section III represents the experimental set up of Body Area Network.Section IV deals with the result analysis and finally in section $\mathrm{V}$ conclusions will be drawn along with the scope for the future work. 


\subsection{Proposed Architecture of Wireless Body Area network:}

In Wireless Body Area Network various physiological parameters are measured by placing sensors on body parts of the patients who are either unable to move (physically challenged) or are under continuous monitoring the patients who don't want to stay in the hospital for longer duration.

A low cost Wireless BAN, using off-the-shelf hardware was built and successfully tested in real time where data was successfully captured and displayed on a website [5]. The BAN collected the pulse rate, the temperature and the location of the patients. The captured data was made available through a graphing application programming interface, where data can be continuously monitored on a website. Future enhancements to safeguard the data, including the encryption of the patient data is under investigation. Currently the BAN is powered using a 9V battery. The sensor node consists of a temperature sensor, a pulse sensor, a ZigBee module, a GPS module and Arduino Uno microcontroller for data processing.

A wide range of ECG recognition algorithms are available, including the wavelet, syntax analysis, mathematical morphology, hidden Markov models, neural networks, fuzzy logic, pattern matching, knowledge-based autoregressive modeling, and support vector machines (SVMs). Data mining and machine learning seems to offer new hope for finding rules in classifications, as with Swift Rule. In fact, computers can't summarize all practical diagnosis rules automatically; human experiences are too complex to be simulated exactly without human computer interaction. However, these methods are insufficient for use in a clinical environment. Our morphology feature- based ECG recognition approach presents a new method that simulates a physician's thinking with regard to diagnosis [2].

Jun et al [8] also describes the architecture of a wearable ECG monitor that is patient location independent and provides continuous monitoring. The signals from the sensors are transmitted using Bluetooth to the smart phone in its area, which can in turn be sent to the destination via internet.

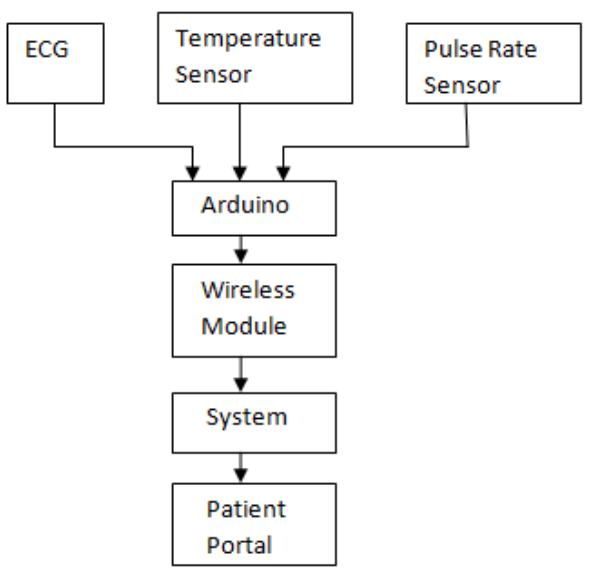

Figure 5: Proposed architecture of Wireless Body Area Network

The proposed architecture as shown in figure 5 represents ECG, temperature and Pulse sensors as various devices placed on body. With the help of arduino the received signals are processed and sent to the doctor's table for future reference. The data is also sent to patient portal also so that they can keep track of their parameters. 
Savita Fet,; Design and Implementation of Wireless Body Area Network using Physiological Parameters. Journal of Biomedical Engineering and Medical Imaging, Volume 4, No 1, February (2017), pp 13-20

\subsection{Experimental Set Up for proposed architecture:}

The experimental set up for the proposed architecture can be broadly classified into Hardware configuration for ECG and software system for the same; hardware configuration for digital temperature sensor and algorithm for pulse heart rate sensor:

\subsubsection{Hardware configuration for ECG:}

To determine the electrical activity of heart; AD8232 Spark fun single Heart rate Monitor board is used. The board gives reading in analog form after continuously recording electrical activity of ECG. As noise is more in ECG; AD8232 acts as Operational amplifier so that clear signal from PR and QT interval can be obtained. For any biopotential and ECG measurements applications AD8232 acts as an integrated signal conditioning block. AD8232 is designed in such a way that even in the presence of noisy conditions caused either by motion or wrong electrode placement user can easily extract, amplify and filter small biopotential signals. [7]

\subsubsection{Software System for ECG}

The software design is classified as

a) For fetching and processing ECG signal and temperature data given by $12 \mathrm{C}$ code is implemented within the Arduino Mega.

b) The other one is a Graphic Unit Interface which is easy to use by the patient. It is developed by using the C\# language under Windows as operating system.

\subsubsection{Hardware configuration for digital temperature sensor:}

The LM32 is a digital temperature sensor having single wire interface which is compatible with National Semiconductor's Sensor Path bus. It measures three temperature zones. LM-32 Sensor-Path data is pulse width encoded, thereby allowing the LM32 to be easily connected to many general purpose microcontrollers.

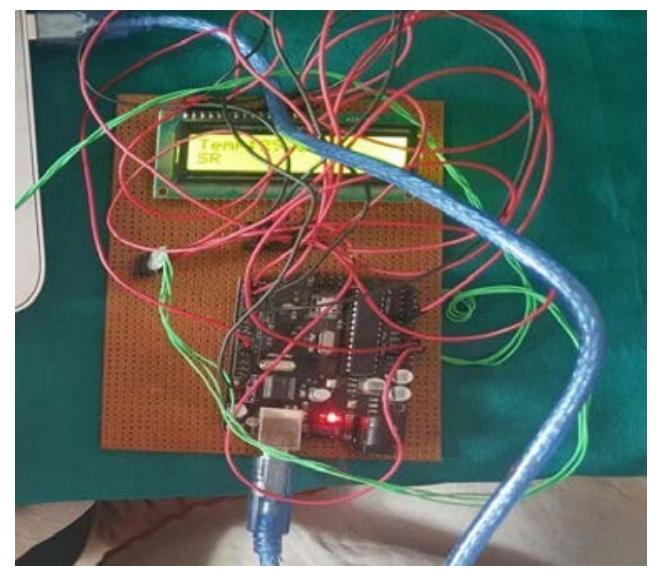

Figure 6: Display of Temperature sensor 


\subsubsection{Algorithm for pulse Heart Rate Monitor:}

Heartbeat and body temperature are very basic and important physiological parameters that are routinely measured whenever a patient arrives in a hospital. To keep track of medical conditions and diagnose disease heart rate monitoring is the primary test to be done by medical professionals [14] .Because of unhealthy eating habits and changed lifestyle Due to sudden changes in lifestyle and unhealthy eating habits, heart and vascular diseases increased in numbers. It affects youth the most [15]. Heart rate is routinely monitored and measured in supervised conditions and background in the clinics. It is a felt need that a system should be designed and implemented in such that the patient will be able to examine and track his/her fitness at their home. This will augment the system performance while offering the advantage of flexibility and maneuverability over existing conventional systems.

After reading ECG signal; distribute the same on the graph. Measure the distance between two R-R peak intervals. Then find pulse heart rate as $1500 /(R-R) \mathrm{mm}$.

\section{Results and discussions:}

From experimental set up the real time patient monitoring portal has been implemented which can sense and diagnose various parameters and send the same over a wireless network through wireless system 802.11.n configuration for maximum data transmission.

1) Temperature was recorded and transmitted to the microcontroller for the processing using LM 32.

2) ECG has been analyzed and filtered using the band pass filter and converted to digital form and then transmitted over the network. It has been analyzed. It was observed that the loss of data was negligible. The ECG signal was thus plotted on patient's phone. There are few precautions one should take while measuring the results:

a) Only 3 patients can be were connected per router.

b) Data is transmitted and analyzed in real time.

c) Filter has to be checked for correct result analysis.

3) Pulse rate sensor determines the same process as the temperature then sends to the pulse rate monitor. Cumulative data thus formed is sent over the network to the doctor's table and patient's portal.

\section{Results}

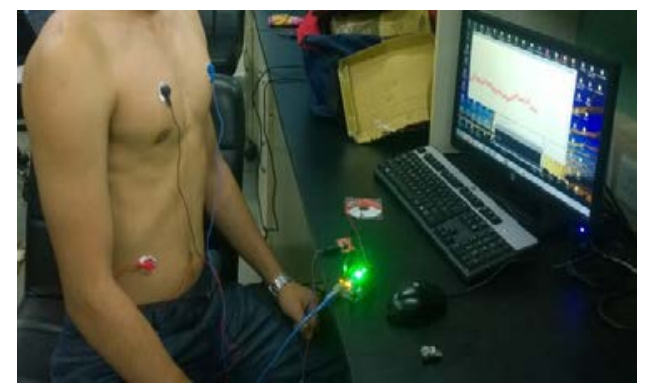

Figure7: ECG testing in real time 
Savita Fet,; Design and Implementation of Wireless Body Area Network using Physiological Parameters. Journal of Biomedical Engineering and Medical Imaging, Volume 4, No 1, February (2017), pp 13-20

The real time testing of ECG is done by connecting the device to the computer. There are three leads which are placed on the body. The circuit is attached with the computer and ECG is taken as shown in figure 7. Result is shown in the figure below (fig.8).

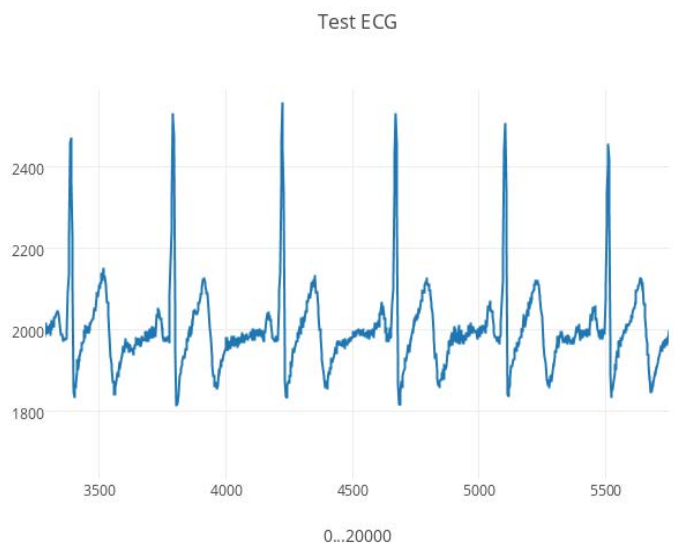

Figure 8:Results of ECG for the patient

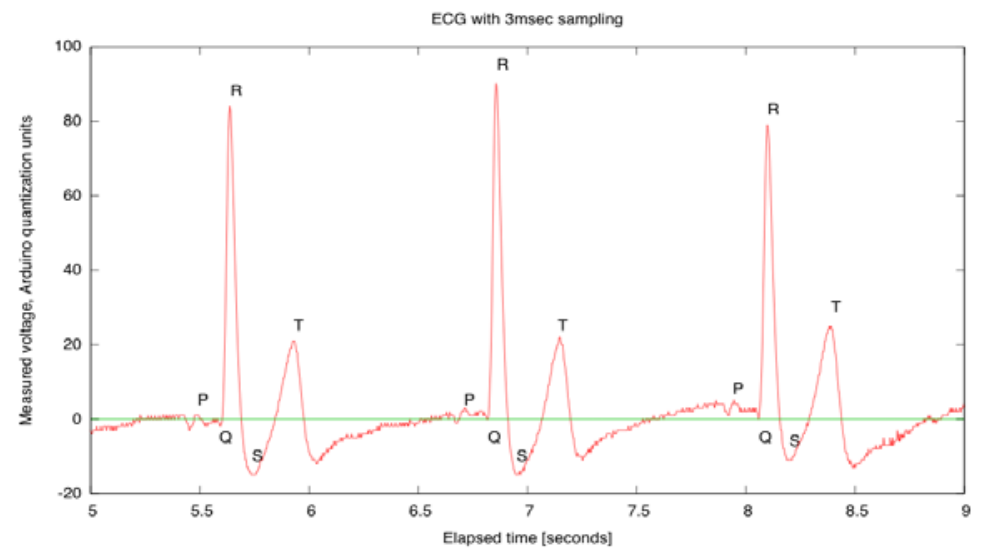

Figure 9: ECG with 3msec Sampling

Figure 9 gives voltage Vs time graph of ECG with $3 \mathrm{msec}$. sampling through which extensive study like for heart rate variability measures, to find the accuracy of R-peak detection etc. can be done.

\section{Future Scope}

In the proposed Wireless Body Area Network three physiological parameters (Body temperature, ECG and Pulse Rate) have been chosen and sensors have been attached to the body. Physiological data had been collected and wirelessly transmitted to the respective locations, either to the doctor or to the patient for further analysis. It can be suggested for future scope that data can be store at cloud and can be accessed anytime, anywhere as and when required.

The WBAN technology can be used for fully assisted computerized physical reformation in itinerant environment and keep a track of the changing parameters during recovery. Designed system can synergize the information from multiple sensors and analyze the data and process the same accordingly. They can be used to send the warnings in the case of extremity, and provide feedback during supervised recovery or any other normal activity. 


\section{REFERENCES}

[1] Nabar, Sidharth, Ayan Banerjee, Sandeep KS Gupta, and Radha Poovendran. "GeMREM:Generative modeldriven resource efficient ecg monitoring in body sensor networks."in Body Sensor Networks (BSN), 2011 International Conference on, pp. 1-6. IEEE, 2011.

[2] 2012 ,A Remote Diagnosis Service Platform for Wearable ECG Monitors 1541-1672/12/\$31.00 (c) INTELLIGENT SYSTEMS Published by the IEEE Computer Society

[3] A.Hussain, N.Alam, K.S.Kwak, 2011 "Directional MAC approach for wireless body area networks," Sensors

[4] Dingjuan Chua ; Pathmasuntharam, J.S. ; Yong Ping Xu WBAN based cableless ECG acquisition system Rui Pan ; Dept. of Electr. \& Comput. Eng., Nat. Univ. of Singapore, Singapore,

[5] Haghani 2013."Implementation of a Wireless Body Area Network for Healthcare Monitoring ", https://www.asee.org/documents/.../5-Haghani-ASEE-Paper-2013.pdf

[6] Xin Liu, Yuanjin Zheng, Myint Wai Phyu, F. N. Endru, V. Navaneethan, and Bin Zhao, March 2012 An UltraLow Power ECG Acquisition and Monitoring ASIC System for WBAN Applications, IEEE Journal on Emerging and Selected Topics in Circuits and Systems, vol. 2, no. 1,

[7] https://www.sparkfun.com/products/12650

[8] DONG Jun, ZHANG Jia-wei1, ZHU Hong-hai1, WANG Li-ping, LIU Xia, LI Zhen-jiang, May, 2014,Wearable ECG Monitors and Its Remote Diagnosis Service Platform, IEEE Intelligent Systems,

[9] P. S. Pandian, K. P. Safeer, P. Gupta, D. T. Shakunthala, B. S. Sundersheshu and V. C. Padaki, Wireless Sensor Network for Wearable Physiological Monitoring, Journal Of Networks, Vol. 3, No. 5, May 2008

[10] Mayuri Navale,Sandhya Damare,Rameshwari Chavan,Renuka Dube,Prof. uhas Patil ,May2014"Android Based Heart Monitoring and Reporting System",International Journal of Advanced Research in Computer and Communication Engineering, Vol. 3, Issue 5

[11] Subhas Chandra Mukhopadhyay (MARCH 2015) "Wearable Sensors for Human Activity Monitoring: A Review "IEEE SENSORS JOURNAL, VOL. 15, NO. 3, 1321

[12] Dheeraj Rathee \& Savita \& S. K. Chakarvarti \&V. R. Singh (September 2014)"Recent trends in Wireless Body Area Network (WBAN) research and cognition based adaptive WBAN architecture for healthcare" Health Technol. , Volume 4, Issue 3, pp 239-244, DOI 10.1007/s12553-014-0083-z

[13] Savita Sindhu,Shruti Vashist and S.K.Chakarvarti March 2016,"Wireless Body Area Network (WBAN): Implementation Techniques: A Review" Communications on Applied Electronics (CAE)- ISSN: 23944714Foundation of Computer Science FCS, New York, USAVolume4-No.7, pp16-20

[14] G E. Billman(2011), "Heart Rate Variability-A Historical Perspective", Frontiers in Physiology., vol. 2, pp. 113.

[15] J.B. Rubin and W.B. Borden (2012), "Coronary Heart Disease in Young Adults", Curr. Atheroscler Rep., vol. 14, no. 2, pp. 140-149. 